\title{
Providing Access: Differences in Pediatric Portal Activation Begin at Patient Check-in
}

Ruth A. Bush, PhD, MPH, FAMIA 1,2,* Vijaya M. Vemulakonda, MD, JD ${ }^{3,4, *}$ Andrew C. Richardson, MS ${ }^{5}$ Sara J. Deakyne Davies, $\mathrm{MPH}^{6}$ George J. Chiang, $\mathrm{MD}^{7,8}$

\footnotetext{
${ }^{1}$ Hahn School of Nursing and Health Science, Beyster Institute for Nursing Research, University of San Diego, San Diego, California, United States

${ }^{2}$ Department of Urology, Rady Children's Hospital San Diego, San Diego, California, United States

${ }^{3}$ Department of Pediatric Urology, Children's Hospital Colorado, Aurora, Colorado, United States

${ }^{4}$ Division of Urology, Department of Surgery, University of Colorado Denver Anschutz Medical Campus, Aurora, Colorado, United States

${ }^{5}$ Clinical Research Informatics, Rady Children's Hospital San Diego, San Diego, California, United States

6 Research Informatics and Analytics, Analytics Resource Center, Children's Hospital Colorado, Aurora, Colorado, United States

7 Department of Urology, University of California San Diego, San Diego, California, United States

8 Rady Children's Institute of Genomic Medicine, Rady Children's Hospital San Diego, San Diego, California, United States
}

Appl Clin Inform 2019;10:670-678.

Address for correspondence Ruth A. Bush, PhD, MPH, FAMIA, Hahn School of Nursing and Health Science, Beyster Institute for Nursing Research, University of San Diego, 5998 Alcala Park; San Diego, CA 92110, United States (e-mail: rbush@sandiego.edu).

\section{Abstract}

Keywords

- meaningful use

- electronic health records and systems

- pediatric health disparities

- health information technology

- patient portal
Background The patient portal interface with individual electronic health records (EHR) was introduced as a tool to enhance participatory medicine. Recent studies suggest adults from racial and ethnic minorities as well as non-English speakers face disproportionate barriers to adoption; however, little data are available for pediatric patients.

Objective The purpose of this study was to examine patient portal offers and activation patterns among pediatric urology patients at two geographically diverse tertiary pediatric hospitals.

Methods Retrospective analysis of 2011 to 2016 electronic portal audit records was conducted among patients aged 18 and younger with at least one outpatient urology clinic visit at two tertiary academic pediatric hospitals and their affiliated networks. Differences in utilization among parents/caregivers and adolescents were examined using multivariate analysis.

Results Of 44,608 individuals seen in a participating urology department during the study period, 21,815 (48.9\%) were offered a code for patient portal activation; of these, 8,605 (19.3\% of total eligible individuals) activated portal access. Logistic regression demonstrated associations between an offer and site $(p<0.001)$, being female $(p<0.001)$, being Asian or white $(p<0.05)$, being non-Hispanic $(p<0.001)$, and reporting English as preferred language $(p<0.001)$. Activating patient portal access

* Co-first coauthors.

received

April 23, 2019

accepted after revision

July 10, 2019 (c) 2019 Georg Thieme Verlag KG Stuttgart · New York
DOI https://doi.org/

10.1055/s-0039-1695792.

ISSN 1869-0327. 
was associated with site $(p<0.001)$, being Asian or white $(p<0.001)$, and reporting English as preferred language $(p<0.001)$.

Conclusion This study found that demographic variations in portal began with demographic differences in which patients were offered an activation code. Fewer than half of those given an access code activated their account. Preferred language, race/ethnicity, and clinic location were associated with likelihood of portal activation. Although patients are increasingly expected to schedule appointments, manage correspondence, request prescription refills, obtain authorizations and referrals, and communicate with the medical team using the portal, this study suggests that in the pediatric specialty setting many patients and caregivers are not offered the opportunity to access these tools.

\section{Background and Significance}

\section{Patient Portal Purpose}

The patient portal (portal) is a secure online Web site that provides patients with 24-hour access to medical information and scheduling tools via the electronic health record (EHR). ${ }^{1-4}$ These tools have been supported by financial incentives provided by the Centers for Medicaid and Medicare Services (CMS) via the Health Information Technology for Economic and Clinical Health (HITECH) Act's Meaningful Use program and, as a result, are generally available in large- and medium-sized hospitals in the United States. ${ }^{5-7}$ The purpose of this access is to empower patients and promote health self-management by providing patients with access to health information, appointment scheduling, bill payment, and secure communication with their health providers outside of face-to-face encounters. ${ }^{8-11}$ The portal may improve patient functional status and reduce high-cost utilization. ${ }^{12}$

\section{Portal Utilization Barriers}

Patient portals continue to be underutilized. ${ }^{1,2,5,8,9,13-16}$ Limited portal familiarity, lack of technological knowledge, low health literacy, and minimal provider endorsement have been linked to low activation, while users are more likely to have more education and more likely to be internet users. ${ }^{9,13,14,17-20}$ Among adult users, personal characteristics such as race/ethnicity, age, gender, education, primary language, and caregiving role influence portal activation; prior studies show that patients of minority race/ethnicity and non-primary English speakers were less likely to activate a portal account. ${ }^{1,5,9,15,21-23}$ Additionally, despite added CMS meaningful use incentives to expand portal use, race, and ethnicity have continued to be independent predictors of portal utilization. ${ }^{16}$

Even when the portal is optimized for a smart phone and accessible using an app, differences in portal use by race and ethnicity remain. ${ }^{24}$ Among adults, it is acknowledged that there are substantive differences in the way portals are promoted among patients, ${ }^{18}$ but systematic reviews and analysis of large datasets have focused on differences associated with activation rates, ${ }^{9,14,16,22}$ but have not evaluated demographic differences in who is offered an activation code, and there is little information about portal recruitment or analysis to examine if differences in portal activation are associated with who is offered an activation code and if this differs by personal and health care delivery factors. Although Irizarry et al reported pediatric caregivers have the most interest in portals, there are few studies or systematic reviews examining pediatric portals. $9,22,25-27$

\section{Objectives}

This study examined patterns of patient portal codes and activation at two tertiary academic pediatric hospitals, one in Colorado and the other in Southern California, by race/ ethnicity, preferred language, patient gender, and activation by caregiver proxy or adolescent patient. One site had both English and Spanish portal information and activation materials and the other provided English-only access. Given past findings within adult patient samples, it was hypothesized that racial/ethnic minority patients, patients with a primary language other than English, and adolescent patients will have lower portal activation rates. Study findings will help researchers and providers understand characteristics of portal activation, adoption, and use.

\section{Methods}

\section{Setting}

The settings were selected to maximize geographic and patient demographic diversity. Site A is a tertiary pediatric hospital with affiliated satellite locations in Colorado. It serves 812,000 children within a seven-state region in the western mountain and plains, with $\sim 6,500$ unique urology outpatient visits annually. Site A's patient racial/ethnic composition is $\sim 82 \%$ white and $17 \%$ Hispanic/Latino. Site B is a tertiary pediatric hospital and its affiliated network, which draws from three counties in Southern California, serving more than 750,000 children, and has $\sim 5,100$ unique urology outpatient visits per year. Site B is located near an international border and its patients' racial/ethnic composition is $\sim 45 \%$ Hispanic/Latino, 35\% white, and 10\% Asian. The Epic Electronic Health Record (EHR) system (Verona, Wisconsin, United States), which incorporates emergency department (ED), inpatient, outpatient 
(including satellite clinics), laboratory, and radiology input into an integrated system, was used at both organizations. Site A has used Epic since 2004 and began portal implementation in 2010. The EHR system has been fully operational at Site B since 2010 with portal (MyChart) introduction in late 2010. At the time of the study, portal functions included the ability to securely message a physician's office, access normal test results, view a child's health summary including immunizations, request prescription refills, request primary-care appointments, and view all scheduled appointments. There were no customizations made to either site's portal other than typical configuration settings at the time of this study.

\section{Patient Portal Activation}

Activation was a two-step process in which clinical staff offered an activation code to caregivers of patients younger than 12 or patients 12 years and older during their office visit allowing them to create a user name and password, verify personal information such as date of birth, and set up security questions. The EHR configuration required the initial registration take place on an internet-connected computer using an up-to-date browser (such as Internet Explorer). Following activation, users could access the portal through an iPhone or Android application, or by computer, with the ability to use secure messaging, view normal test results, look at abbreviated health summaries including problem lists, after visit summaries, and patient instructions; request prescription refills; request primary care appointments; and view scheduled appointments. Pediatric patients aged 12 to 17, with parental permission, could activate their own accounts; in these cases, parents were allowed proxy access to limited information such as their child's immunization list, but could not view the problem list, medications, allergies, upcoming appointments, or released laboratory tests. The proxy mode allows for protection of confidential communication between provider and patient about topics such as family planning, sexually transmitted infections, and pregnancy.

Enrollment materials, portal log-in screen, and portal functions such as secure messaging were available in English at Site A and in both English and Spanish at Site B. Interpretation services were available for those needing assistance in other languages. There was no standard approach to patient portal introduction at the time of the study; introduction and discussion of portal adoption workflow varied by site. Site A held "Open Houses" where providers and staff could see a portal demonstration and ask questions. Training was conducted during departmental meetings, including nurses and medical assistants, who introduced patients and their families to the potential utility of portal use at the time that patients were roomed in the outpatient clinic; caregivers and patients were allowed to use the exam room computer to activate their accounts. Volunteers were also present at MyChart kiosks in the first half of 2011 to assist and encourage people to activate their accounts. Site B provided training to the patient access representatives who introduced the portal functions during patient check-in and provided computers in both primary care and specialty practice waiting rooms to facilitate same-day activation.

\section{Patients}

Patients were included if they had at least one outpatient urology visit from 2011 through 2016 and were aged 2 to 18 years at the time of visit. By including those patients older than 2, most circumcision were excluded and diagnoses where a patient/caregiver or adolescent patient were likely to look up laboratory results and wish to communicate electronically with their providers were included. At both sites, the pediatric urology division is the primary referral provider for an expansive geographic area and manages care for children with chronic diseases, creating a large racially/ethnically diverse and multilingual patient sample of more than 40,000 in which to examine portal activation patterns.

\section{Data Source}

After obtaining Institutional Review Board approval, with a waiver of consent, activation of the patient portal was retrospectively assessed from January 2011 to May 2016 for all patients with at least one visit with an outpatient urology visit using activation audit data. Demographic variables extracted included the patient's gender, preferred language, race, and ethnicity. The patient's site was also captured. For those individuals who activated an account, date of birth, and date of activation were used to assign individuals to an age group. Among those who were not offered a code or did not activate an account, those who were at least 11 years old at the start of the study period were classified as adolescents. Those who were younger than 11 years during the time period were classified as children. While age at first appointment was also considered, using this calculation over-represented children who also had the opportunity to enroll as teenagers.

\section{Measures}

For this analysis, "Offered" was defined as having an audit record of offering a patient the opportunity to create a patient portal account, regardless of whether the offer was accepted or declined. "Activated" was defined as having an active account or deactivated account at the time of data extraction. Patients who received but did not activate a code were classified as not activated.

\section{Statistical Analysis}

Summary statistics were calculated to describe the sample being studied. Associations among categorical demographic variables, being offered a code, and activating portal access were analyzed using Pearson's chi-square $\left(x^{2}\right)$. Those bivariate associations with a $p$-value of less than 0.05 were entered in a two-binary logistic regression model in which all the independent variables were entered in a single, simultaneous block. The first model examined the variable relationships with offer (offered/not offered) and the second with activation (activated/not activated) status. Missing data were coded as no response and included as a category in the analysis. Analyses were performed using IBM SPSS Statistics for Windows, Version 25 (Armonk, New York, United States: IBM Corp). 
Table 1 Offer and activation by site

\begin{tabular}{|c|l|l|l|l|}
\hline & \multicolumn{2}{|l|}{ Site A } & \multicolumn{2}{l|}{ Site B } \\
\hline Code not offered & 14,404 & $55.9 \%$ & 8,389 & $44.5 \%$ \\
\hline Code offered & 11,341 & $44.1 \%$ & 10,473 & $55.5 \%$ \\
\hline Activated & 3,741 & $33.0 \%$ & 4,864 & $46.4 \%$ \\
\hline Deactivated & 515 & $4.5 \%$ & 2,223 & $21.2 \%$ \\
\hline Code not activated & 7,085 & $62.5 \%$ & 3,386 & $32.3 \%$ \\
\hline Total records & 25,745 & & 18,862 & \\
\hline
\end{tabular}

\section{Results}

\section{Characteristics of Patients Offered a Code}

Of 44,608 individuals seen in a participating urology department during the study period, 21,815 or slightly fewer than half (48.9\%) were offered a code for portal activation ( - Table 1$)$. Chi-squared analysis demonstrated significant differences (all $p$-values $<0.001$ ) between offer status and the site, gender, race, ethnicity, and preferred language (-Table 2). Of those individuals who did not report race in the EHR, 26\% were offered a code; $38 \%$ of those who reported Spanish as their preferred language were offered a code. All the demographic variables were entered in a bivariate logistic regression. The model had an omnibus $\chi^{2}$ of $1,871.45$ with a $p$-value of $<0.001$; a log-likelihood of 59,942.81 and Nagelkerke's $R^{2}$ of 0.06 . All entered variables contributed significantly to the model (-Table 3 ). The strongest association with offered code was site, with patients at Site B almost twice as likely to be offered a code (odds ratio [OR]: $1.85 ; p<0.001$ ). Within the categories of race, individuals not having race recorded were a third as likely to be offered a code (OR: $0.34 ; p<0.001$ ) compared with the reference group of white and those who were recorded as other were only $80 \%$ as likely as whites (OR: $0.79(p<0.001)$. Those who reported their primary language as Spanish or a language neither Spanish nor English were almost 40\% less likely to have been offered a code to activate the portal compared with those who reported English as preferred language.

Age group data were available for 25,758 of the 44,608 sample (58\%). Using age overrepresented those who had been offered a code and underrepresented those for whom race and ethnicity were not available. A separate analysis for those aged 2 to 11 and those 12 and older was conducted. Within this subsample, race, ethnicity, gender, language, and site were significant in both children and adolescents. Children who did not report race or who reported race as other $\left(x^{2}=236.85 ; p<0.0001\right)$, those who did not report an ethnicity $\left(x^{2}=322.58 ; p<0.0001\right)$, those who reported Spanish as their primary language $\left(\chi^{2}=373.09 ; p<0.0001\right)$, and male patients $\left(x^{2}=49.27 ; p<0.0001\right)$ were significantly less likely to be offered a code. Site was also significant $\left(\chi^{2}=643.17\right.$;

Table 2 Factors associated with offer and activation

\begin{tabular}{|c|c|c|c|c|c|c|c|c|}
\hline & $\begin{array}{l}\text { Offered } \\
(n=21,815 \\
[48.9 \%])\end{array}$ & $\begin{array}{l}\text { Not offered } \\
(n=22,793)\end{array}$ & \multirow[t]{2}{*}{$x^{2}$} & \multirow[t]{2}{*}{$p$-Value } & \multirow{2}{*}{$\begin{array}{l}\text { Activated } \\
(n=11338 \\
[52.0 \%]) \\
\text { Frequency }(\%)\end{array}$} & \multirow{2}{*}{$\begin{array}{l}\text { Not activated } \\
(n=10476)\end{array}$} & \multirow[t]{2}{*}{$x^{2}$} & \multirow[t]{2}{*}{$p$-Value } \\
\hline & Frequency (\%) & Frequency (\%) & & & & & & \\
\hline Site & & & & & & & & \\
\hline Site A & $1,1341(52.0)$ & $14,404(63.2)$ & 573.5 & $<0.001$ & 4,255 (37.5) & 7,086 (67.6) & $1,977.9$ & $<0.001$ \\
\hline Site B & $10,473(48.0)$ & $8,389(36.8)$ & & & $7,083(62.5)$ & $3,390(32.4)$ & & \\
\hline Sex & & & & & & & & \\
\hline Female & $7,560(34.7)$ & 6,779 (29.7) & 123.3 & $<0.001$ & $3,886(34.3)$ & $3,674(35.1)$ & 1.5 & 0.217 \\
\hline Male & $14,254(65.3)$ & $16,012(70.2)$ & & & $7,452(65.7)$ & $6,802(64.9)$ & & \\
\hline Race & & & & & & & & \\
\hline White & $12,436(57.0)$ & $11,218(49.2)$ & 775.0 & $<0.001$ & $6,483(57.2)$ & $5,953(56.8)$ & 117.3 & $<0.001$ \\
\hline Asian & $762(3.5)$ & $591(2.6)$ & & & $484(4.3)$ & $278(2.7)$ & & \\
\hline Black & $1,028(4.7)$ & $1,020(4.5)$ & & & $473(4.2)$ & $555(5.3)$ & & \\
\hline Other & $6,858(31.4)$ & 7,896 (34.6) & & & $3,623(32.0)$ & 3,235 (30.9) & & \\
\hline Not reported & $731(3.4)$ & 2,068 (9.1) & & & $275(2.4)$ & $455(4.3)$ & & \\
\hline Ethnicity & & & & & & & & \\
\hline Hispanic & $7,384(33.8)$ & $8,653(38.0)$ & 625.7 & $<0.001$ & $7,306(64.4)$ & $6,351(60.6)$ & 83.4 & $<0.001$ \\
\hline Non-Hispanic & $13,657(62.6)$ & $12,248(53.7)$ & & & $3,743(33.0)$ & $3,641(34.8)$ & & \\
\hline Not Reported & 773 (3.5) & $1,892(8.3)$ & & & $289(2.5)$ & $484(4.6)$ & & \\
\hline Language & & & & & & & & \\
\hline English & $18,785(86.1)$ & $17,877(78.4)$ & 451.0 & $<0.001$ & 9,835 (86.7) & $8,950(85.4)$ & 25.4 & $<0.001$ \\
\hline Spanish & $2,628(12.0)$ & 4,205 (18.4) & & & $1,265(11.2)$ & $1,363(13.0)$ & & \\
\hline Other & $402(1.8)$ & $711(3.1)$ & & & $238(2.1)$ & $163(1.6)$ & & \\
\hline
\end{tabular}


Table 3 Binary logistic regression

\begin{tabular}{|c|c|c|c|c|c|c|c|c|c|}
\hline \multicolumn{5}{|l|}{ Offered } & \multicolumn{5}{|l|}{ Activated } \\
\hline & \multirow[t]{2}{*}{ Odds ratio } & \multirow[t]{2}{*}{$p$-Value } & \multicolumn{2}{|c|}{$\begin{array}{l}95 \% \mathrm{Cl} \text { for EXP } \\
\text { (B) }\end{array}$} & & \multirow[t]{2}{*}{ Odds ratio } & \multirow[t]{2}{*}{$p$-Value } & \multicolumn{2}{|c|}{$\begin{array}{l}95 \% \mathrm{Cl} \text { for EXP } \\
\text { (B) }\end{array}$} \\
\hline & & & Lower & Upper & & & & Lower & Upper \\
\hline Site $A$ & 0.54 & $<0.001$ & 0.52 & 0.56 & Site $\mathrm{A}$ & 0.26 & $<0.001$ & 0.24 & 0.28 \\
\hline Female & 1.27 & $<0.001$ & 1.22 & 1.32 & Female & 1.06 & 0.03 & 1.01 & 1.14 \\
\hline Race $^{a}$ & & $<0.001$ & & & Race $^{a}$ & & $<0.001$ & & \\
\hline Not reported & 0.34 & $<0.001$ & 0.30 & 0.38 & Not reported & 0.54 & $<0.001$ & 0.43 & 0.67 \\
\hline Other & 0.79 & $<0.001$ & 0.75 & 0.83 & Other & 0.81 & $<0.001$ & 0.75 & 0.87 \\
\hline Black & 0.92 & 0.081 & 0.84 & 1.01 & Black & 0.73 & 0.081 & 0.63 & 0.83 \\
\hline Asian & 1.06 & 0.301 & 0.95 & 1.19 & Asian & 1.04 & 0.301 & 0.95 & 1.19 \\
\hline Ethnicity $^{\mathrm{b}}$ & & $<0.001$ & & & Ethnicity $^{\mathrm{b}}$ & & $<0.001$ & & \\
\hline Not reported & 0.78 & $<0.001$ & 0.70 & 0.88 & Not reported & 0.89 & 0.28 & 0.72 & 1.10 \\
\hline Hispanic & 0.97 & 0.336 & 0.92 & 1.03 & Hispanic & 0.85 & $<0.001$ & 0.79 & 0.92 \\
\hline Language $^{c}$ & & $<0.001$ & & & Language $^{c}$ & & $<0.001$ & & \\
\hline Other & 0.57 & $<0.001$ & 0.50 & 0.65 & Other & 0.99 & 0.97 & 0.80 & 1.24 \\
\hline Spanish & 0.61 & $<.001$ & 0.57 & 0.65 & Spanish & 0.76 & $<0.001$ & 0.69 & 0.84 \\
\hline Constant & 1.63 & $<0.001$ & & & Constant & 0.73 & $<0.001$ & & \\
\hline
\end{tabular}

${ }^{a}$ White as reference group.

${ }^{b}$ Non-Hispanic as reference group.

${ }^{c}$ English as reference group.

$p<0.0001$ ) as children at Site B were more likely to be offered a code. Among teens, those who had not reported a race were significantly less likely to be offered a code and those who identified as white were more likely to be offered a code $\left(x^{2}=163.03 ; p<0.0001\right)$; those who did not report an ethnicity $\left(\chi^{2}=128.00 ; p<0.0001\right)$; those who reported Spanish as their primary language $\left(\chi^{2}=71.68 ; p<0.0001\right)$; and female patients were more likely to be offered a code $\left(\chi^{2}=40.31 ; p<0.0001\right)$. Site was also significant $\left(x^{2}=7.64 ; p<0.006\right)$ as teens at Site B were more likely to be offered a code. In children with a parent/ caregiver proxy, caregiver gender could not be assessed.

\section{Characteristics of Patients Who Activated Portal Access}

To explore characteristics associated with activation, the 11,338 activators (25.4\% of total eligible individuals) were compared with the 10,476 nonactivators ( - Table 1 ). Chi-squared analysis demonstrated highly significant differences $(p<0.001)$ between activation status and site, race, gender, and preferred language (-Table 2). The bivariate logistic model had an omnibus $\chi^{2}$ of $1,662.34$ with a $p$-value of $<0.001$; a log-likelihood of 27,599.23 and Nagelkerke's $R^{2}$ of 0.10 ( - Table 3). All of the entered variables contributed significantly to the model. Site B had the strongest association with activation (OR: 3.85; $p<0.001$ ), with those at Site B almost four times as likely to activate their account. Those who reported their preferred language as Spanish were only $76 \%$ as likely to activate their account as those who reported English (OR: 0.76; $p<0.001$ ). Those who did not report a race (OR: $0.54 ; p<0.001$ ), reported other (OR: $0.81 ; p<0.001$ ), or reported being black (OR: 0.73 ; $p<0.001$ ) were less likely to activate the portal compared with whites. Of note, 14,754 individuals' (33\%) race was captured as other or multiracial, indicating current available measurement categories do not match patient self-identification. A separate analysis of activation by age was conducted. Within those patients aged 2 to 11 years, being white $\left(x^{2}=29.51\right.$; $p<0.0001)$, non-Hispanic $\left(\chi^{2}=9.18 ; p=0.01\right)$, English as primary language $\left(x^{2}=55.26 ; p<0.0001\right)$, and Site $\mathrm{B}$ $\left(x^{2}=353.83 ; p<0.0001\right)$ were significantly associated with activation. Patient gender was not significant. Similarly, in adolescents being white $\left(x^{2}=38.59 ; p<0.0001\right)$, non-Hispanic $\left(x^{2}=14.07 ; p<0.0009\right)$, female $\left(x^{2}=212.31 ; p<0.0001\right)$, and Site B $\left(\chi^{2}=679.87 ; p<0.0001\right)$ were significantly associated with activation. Primary language was not significant among the teens.

\section{Conclusion}

Within this large, geographically and demographically diverse patient sample, pediatric patients of minority race, ethnicity, and reported primary language other than English were significantly less likely to be offered a code and to activate patient portal access. These differences remained when looking specifically at children's accounts activated by caregivers and those activated by adolescents. Although there are many studies reporting user volume and demographics of patient portal users within adult samples, few studies have evaluated demographic differences in who is offered a portal access 
code. ${ }^{1,23}$ This study expands existing adult literature on portal activation to the pediatric population and addresses the current gap in the literature by evaluating disparities in who is offered portal access.

The primary finding of this study was that fewer than $50 \%$ of patients seen in the outpatient clinic setting were offered a portal code. Previous portal studies have identified potential barriers to using portals, including computer access, primary language other than English, and lack of information about the portal and its potential utility, with these barriers heightened in health systems serving less advantaged populations. ${ }^{9,14,16,17}$ Notably, one pediatric study found being of Hispanic ethnicity decreased portal activation by $\sim 25 \%{ }^{28}$ This study demonstrates that, in addition to these previously described barriers to utilization, demographic factors may impact the likelihood of patients being informed and offered access to the patient portal, suggesting that underlying biases within the health care system may disproportionately limit the ability of minority and non-English-speaking patients and their caregivers to create a patient portal account, a necessary first step for portal activation and utilization.

A second finding of this study was that, among those who did receive an access code for the portal, less than half (45\%) activated the portal. Portal activation was highly associated with site of care, race, ethnicity, and preferred language. Language appeared to be the most significant barrier to activation, with those reporting Spanish as a preferred language one-third as likely to activate portal usage when controlling for other demographic factors. Moreover, Arabic $(n=286)$, Somali $(n=141)$, Vietnamese $(n=97)$, and Chinese $(n=70)$ were also represented within this population and were only half as likely to activate the portal compared with primary English speakers. Although portal information and log-in interface were available in English and Spanish at one site, nearly all available medical records and test results in the portal were presented in English, suggesting decreased benefit of utilization among non-English-speaking populations. These findings are in congruence with the adult literature, where the issue of language as a barrier to portal use has been noted. ${ }^{5,8,17,20}$ It is noteworthy that the site that provided bilingual portal information did have higher offer and activation rates among those who were not primarily English speakers.

This study did not directly address the association of socioeconomic status with patient portal utilization, but did find that demographic factors such as race, ethnicity, gender, and location also impacted patient portal utilization. These findings suggest that demographic factors beyond language may impact portal utilization. Prior studies have shown that portal utilization may be impeded by limited health and electronic literacy among those with lower income level and educational attainment, which has been characterized as the "digital divide."23,29-32 Portal tasks require more health literacy and familiarity with technology than has been asked of patients previously; yet, it is unclear that the health system is addressing this gap in understanding.

Limited portal adoption and the associated disparate impact on minority and non-English-speaking patients are concerning due to the underlying expectation that patients will utilize electronic communication modalities such as the patient portal to manage their health care. Patients are expected to schedule appointments, manage correspondence, request prescription refills, obtain authorizations and referrals, and communicate with the medical team using the portal. ${ }^{11,33,34}$ The portal not only allows patients to track their health and increase their knowledge but also to provide information and to raise questions and concerns with their providers outside of the office setting. Such use creates a feedback loop allowing providers to know how their patients are doing and to improve efficiency during clinic visits. ${ }^{35}$ In the absence of effective portal utilization, providers and patients may be less likely to gain the information they need, which may lead to health treatment delay and potentially to an increased risk of poor health outcomes.

Both health systems in this study engaged in portal recruitment efforts by enlisting existing clinic and administrative personal, such as those checking-in patients or rooming patients for the appointments, to discuss enrollment for portal access. Clinicians were encouraged to discuss the benefits of the portal with patients during clinic visits. Additionally, clinical workflows were modified and computers installed in waiting rooms and individuals at one site allowed to use computers in exam rooms to allow more opportunities to sign up for the portal while needed activation details were fresh in patient and caregivers' minds. Providing computers, which were necessary for initial activation, suggests an implicit recognition that, while most patients and caregivers have access to smartphones, many potential users may not have home computer access. ${ }^{19,21,30}$ This process also suggests informal recognition of potential electronic and health literacy barriers to activation that may be ameliorated by access to clinical personnel at the time of activation, despite the lack of formal instruction in the technological skills needed to support effective portal use, which is similar to benefit of individually based interventions found in adult studies. ${ }^{26}$ Being part of an integrated health system with integrated primary and specialty care likely raises the patient or proxy's view of portal activation utility.

This study highlights existing barriers to "meaningful use" initiatives such as the patient portal across diverse patient populations. Although data on patient portal use in pediatrics are limited, prior studies suggest that the HITECH-funded incentive program for meaningful use of the EHR has been less impactful in the pediatric setting and that meaningful use metrics may be seen as less relevant to pediatric care. ${ }^{36,37}$ To achieve equal access and support a wide variety of patients, additional research is needed to clarify current barriers to utilization of this technology. Additionally, thoughtful dissemination and implementation strategies for health information technology such as the patient portal should expand to address current gaps in health and technologic knowledge, both through improved structured instruction within the health system, as well as more innovative strategies for engagement through community organizations. Finally, improved understanding of patient access to electronic resources such as computers and smartphones may facilitate alternative methods such as blended online-offline interventions to expand and to improve the quality of access. ${ }^{38,39}$ 


\section{Limitations}

This study leveraged existing EHR data collected from embedded data fields in a shared EHR platform across diverse health systems to allow for more robust evaluation of practice patterns related to patient portal dissemination and implementation. While use of this methodology allowed for a more efficient data capture process compared with traditional chart review, this process also led to several imitations. First, data were limited by what was collected via the EHR. While we were able to obtain general data regarding patient portal sign-up procedures, clinical workflows, and portal marketing materials, we were unable to collect more specific data regarding patient-staff or patientclinician interactions related to portal education and activation, data regarding how the caregiver or patient received an access code, or who actually activated the account (patient, caregiver, or clinic personnel). Consequently, potential differences in clinic site approach between the two sites could not be incorporated in the multivariate model. Additionally, we do not know the impact of specialty versus primary care recruitment or if there were interactions in portal interest and acuity of condition and integration in the health system. Second, we utilized existing limited-use data sharing agreements to facilitate multicenter data collection. Due to the limitations of these agreements, although birth date was used for data extraction and age at activation could be calculated, age could not easily be calculated for those patients who did not activate portal access and ability to compare children with a parent proxy to adolescents was limited. As a result, we were unable to assess the association of patient age with likelihood of approach or portal activation within a large subset of our sample. We also did not have proxies for sociodemographic status such as education, insurance, or residential zip code.

Third, unlike the adult population, the majority of individuals activating the portal in the pediatric setting are the parent caregivers of children under the age of 12 . Little is known about how this proxy relationship may change the perception of portal usefulness or willingness to activate. While there were data about patient gender, there was no information about the gender of parent/caregivers. Many of the users may have been mothers, which would be congruent with more females being portal users; however, this information was not readily available in this extracted dataset and should be explored further. We were not able to track the temporal relationship of activation potentially changing over time as the portal ceases to be a new and unknown technology. Finally, patient health severity and health system utilization could not be quantified to allow for assessment of the impact of patient disease state on portal adoption. One hypothesis is that those individuals and families with more frequent healthcare utilization might be more likely to utilize the portal due to the increased benefit of accessing visit scheduling, health information, and provider communication in one place; further studies are needed to understand the relationship of health care utilization and disease severity with portal activation. Despite these limitations, this study provides new and important information about differences in patient portal code offers as well as potential demographic factors limiting portal activation in the pediatric setting and provides insights to direct future investigations regarding potential barriers to patient portal adoption.

\section{Implications}

EHR audit data of almost 45,000 individuals from two pediatric healthcare systems in Colorado and California demonstrated fewer than $50 \%$ of those with an outpatient appointment were offered a code to access the portal and fewer than $25 \%$ of all eligible individuals activated portal access. As previously described in adults, pediatric patients of minority race/ethnicity and who were primarily nonEnglish speaking in this study were less likely to be offered or to activate their access to the patient portal.

Limited portal adoption is concerning because of growing institutional prioritization of electronic patient engagement. Patients are increasingly expected to schedule appointments, access health information, request prescription refills, obtain authorizations and referrals, and communicate with the medical team using the portal. This study suggests that current level of patient engagement with electronic technology such as the patient portal is inadequate to meet these expectations.

Low offer rates may suggest implicit biases in the health system regarding which patients would benefit from patient portal access. Furthermore, low activation rates may suggest parents and patients do not understand the potential role of the portal in improving the quality of their health care. Further understanding of technical and social barriers to patient engagement and utilization of technology and the potential disparate effect on minority and non-Englishspeaking patients is essential. Meeting the complexities of these issues will require exploring translatable records, supporting health literacy, increasing technological familiarity, and improving internet and mobile data access. Additionally, engagement of patients and families to ensure that the portal meets their needs and values is essential to optimize the potential utility of the patient portal.

\section{Clinical Relevance Statement}

Meaningful use metrics such as electronic access to patient records have led to increased expectations for patients and caregivers to utilize health technology to make appointments, track laboratory results, and communicate with their providers outside of the clinic visit. This study was able to compare a sample with English/Spanish portal recruitment materials with one whose materials were English-only and suggests that the impact of tools such as the patient portal on patient care is limited by lack of patient adoption, with barriers to adoption disproportionately affecting racial/ ethnic minorities and non-English-speaking patients. As a result, efforts to address underlying limitations in existing patient-oriented technologies, such as language limitations, health literacy, and technologic access, are needed to optimize the potential impact of meaningful use mandates on patient engagement and outcomes. 


\section{Multiple Choice Questions}

1. What is the most important focus of future studies regarding the moderate rate of portal adoptions in pediatric patients?

a. Understand why interest is not uniform among all potential users.

b. Explore how individuals meaningfully utilize health information technology.

c. How patients utilize technology.

d. Focus on the providers' perspective of patient portal use.

Correct Answer: The correct answer is option b. Future studies that include patient and caregiver health literacy, education level, socioeconomic status, household size, and family structure are planned to augment current knowledge about barriers to patient portal utilization and to transform patient portal utilization from a meaningful use requirement into a meaningful experience for the patient and healthcare team.

2. Which statement is most accurate in describing portal activation in this study?

a. The majority of those given access code activated and used their account.

b. Gender was associated with both being approached and activating portal accounts.

c. Patient portal access was associated with clinical site.

d. Pediatric populations are different from adults in portal activation patterns.

Correct Answer: The correct answer is option c. As previously described in adults, pediatric patients of minority race/ethnicity were less likely to be approached or to activate patient portal access in this study. The majority of those given an access code did not activate their account, which is similar to findings in adults. A difference by site was the most statistically significant association. Further understanding of technical and social barriers to patient engagement and utilization of technology is needed to optimize the utility of the patient portal as a patient engagement.

\section{Protection of Human and Animal Subjects}

This study was performed in compliance with the World Medical Association of Helsinki on Ethical Principles for Medical Research Involving Human Subjects and was reviewed by the University of California, San Diego, and University of Colorado Institutional Review Boards.

\section{Funding}

This project was supported in part by the Agency for Healthcare Research and Quality (grant numbers R00 HS022404 [R.A.B.] and K08 HS024597-01 [V.M.V.] as well as by the American Urological Association Rising Stars in Urology Research Award Program and the Frank and Marion Hinman Urology Research Fund (V.M.V.). The content is solely the responsibility of the authors and does not necessarily represent the official views of the Agency for Healthcare Research and Quality, the American Urological Association, or the Frank and Marion Hinman Urology Research Fund.

\section{Conflict of Interest}

None declared.

\section{References}

1 Griffin A, Skinner A, Thornhill J, Weinberger M. Patient portals: who uses them? What features do they use? And do they reduce hospital readmissions?. Appl Clin Inform 2016;7(02):489-501

2 Mendu ML, Waikar SS. Electronic health record patient portals in CKD and hypertension management: meaningfully used? Clin J Am Soc Nephrol 2015;10(11):1897-1899

3 Nguyen Q, Bartlett G, Rodriguez C, Tellier P-P. Young adults on the perceived benefits and expected use of personal health records: a qualitative descriptive study.J Innov Health Inform 2016;23(01):171

4 Redelmeier DA, Kraus NC. Patterns in patient access and utilization of online medical records: analysis of MyChart. J Med Internet Res 2018;20(02):e43

5 Lyles CR, Fruchterman J, Youdelman M, Schillinger D. Legal, practical, and ethical considerations for making online patient portals accessible for all. Am J Public Health 2017;107(10):1608-1611

6 Percent of Hospitals, By Type, that Possess Certified Health IT. Available at: /quickstats/pages/certified-electronic-health-record -technology-in-hospitals.php. Accessed March 5, 2019

7 Shaw CL, Casterline GL, Taylor D, Fogle M, Granger B. Increasing health portal utilization in cardiac ambulatory patients: a pilot project. Comput Inform Nurs 2017;35(10):512-519

8 Irizarry T, Shoemake J, Nilsen ML, Czaja S, Beach S, DeVito Dabbs A. Patient portals as a tool for health care engagement: a mixedmethod study of older adults with varying levels of health literacy and prior patient portal use. J Med Internet Res 2017;19(03):e99

9 Irizarry T, DeVito Dabbs A, Curran CR. Patient portals and patient engagement: a state of the science review. JMed Internet Res 2015;17(06):e148

10 Sieck CJ, Hefner JL, Schnierle J, et al. The rules of engagement: perspectives on secure messaging from experienced ambulatory patient portal users. JMIR Med Inform 2017;5(03):e13

11 Jilka SR, Callahan R, Sevdalis N, Mayer EK, Darzi A. "Nothing About Me Without Me": an interpretative review of patient accessible electronic health records. J Med Internet Res 2015;17(06):e161

12 Sorondo B, Allen A, Fathima S, Bayleran J, Sabbagh I. Patient portal as a tool for enhancing patient experience and improving quality of care in primary care practices. EGEMS (Wash DC) 2017;4(01):1262

13 Chang E, Blondon K, Lyles CR, Jordan L, Ralston JD. Racial/ethnic variation in devices used to access patient portals. Am J Manag Care 2018;24(01):e1-e8

14 Fix GM, Hogan TP, Amante DJ, McInnes DK, Nazi KM, Simon SR. Encouraging patient portal use in the patient-centered medical home: three stakeholder perspectives. J Med Internet Res 2016;18 (11):e308

15 Goel MS, Brown TL, Williams A, Hasnain-Wynia R, Thompson JA, Baker DW. Disparities in enrollment and use of an electronic patient portal. J Gen Intern Med 2011;26(10):1112-1116

16 Turner K, Hong Y-R, Yadav S, Huo J, Mainous AG. Patient portal utilization: before and after stage 2 electronic health record meaningful use. JAm Med Inform Assoc 2019:ocz030

17 Ackerman SL, Sarkar U, Tieu L, et al. Meaningful use in the safety net: a rapid ethnography of patient portal implementation at five community health centers in California. JAm Med Inform Assoc 2017;24(05):903-912

18 Ancker JS, Osorio SN, Cheriff A, Cole CL, Silver M, Kaushal R. Patient activation and use of an electronic patient portal. Inform Health Soc Care 2015;40(03):254-266 
19 McCloud RF, Okechukwu CA, Sorensen G, Viswanath K. Beyond access: barriers to internet health information seeking among the urban poor. JAm Med Inform Assoc 2016;23(06):1053-1059

20 Tieu L, Sarkar U, Schillinger D, et al. Barriers and facilitators to online portal use among patients and caregivers in a safety net health care system: a qualitative study. J Med Internet Res 2015;17(12):e275

21 Jhamb M, Cavanaugh KL, Bian A, et al. Disparities in electronic health record patient portal use in nephrology clinics. Clin J Am Soc Nephrol 2015;10(11):2013-2022

22 Showell C. Barriers to the use of personal health records by patients: a structured review. Peer] 2017;5:e3268

23 Ancker JS, Nosal S, Hauser D, Way C, Calman N. Access policy and the digital divide in patient access to medical records. Health Policy Technol 2017;6(01):3-11

24 Graetz I, Huang J, Brand RJ, Hsu J, Yamin CK, Reed ME. Bridging the digital divide: mobile access to personal health records among patients with diabetes. Am J Manag Care 2018;24(01):43-48

25 Bush RA, Richardson AC, Cardona-Grau D, Din H, Kuelbs CL, Chiang GJ. Patient portal usage in pediatric urology: is it meaningful use for everyone? Urol Pract 2018;5(04):279-285

26 Grossman LV, Masterson Creber RM, Benda NC, Wright D, Vawdrey DK, Ancker JS. Interventions to increase patient portal use in vulnerable populations: a systematic review. JAm Med Inform Assoc 2019. Doi: 10.1093/jamia/ocz023. [Epub ahead of print]

27 Thompson LA, Martinko T, Budd P, Mercado R, Schentrup AM. Meaningful use of a confidential adolescent patient portal. J Adolesc Health 2016;58(02):134-140

28 Ketterer T, West DW, Sanders VP, Hossain J, Kondo MC, Sharif I. Correlates of patient portal enrollment and activation in primary care pediatrics. Acad Pediatr 2013;13(03):264-271

29 Kimball AB, Corey KC, Kvedar JC. Engaging patients to decrease costs and improve outcomes. Med Econ 2015;92(02):36-37
30 Bush RA, Barlow H, Pérez A, Vazquez B, Mack J, Connelly CD. Internet access influences community clinic portal use. Health Equity 2018;2(01):161-166

31 Featherall J, Lapin B, Chaitoff A, Havele SA, Thompson N, Katzan I. Characterization of patient interest in provider-based consumer health information technology: survey study. J Med Internet Res 2018;20(04):e128

32 Niemi A, Hupli M, Koivunen M. The use of electronic communication for patient-professional interaction - nursing staff's point of view. Finn J EHealth EWelfare 2016;8(04):200-215

33 Coughlin SS, Prochaska JJ, Williams LB, et al. Patient web portals, disease management, and primary prevention. Risk Manag Healthc Policy 2017;10:33-40

34 Voruganti T, Grunfeld E, Makuwaza T, Bender JL. Web-based tools for text-based patient-provider communication in chronic conditions: scoping review. J Med Internet Res 2017;19(10):e366

35 Kruse CS, Argueta DA, Lopez L, Nair A. Patient and provider attitudes toward the use of patient portals for the management of chronic disease: a systematic review. J Med Internet Res 2015;17(02):e40

36 Nakamura MM, Harper MB, Castro AV, Yu FB Jr, Jha AK. Impact of the meaningful use incentive program on electronic health record adoption by US children's hospitals. J Am Med Inform Assoc 2015; 22(02):390-398

37 Teufel RJ II, Yu FB Jr, Nakamura MM, Harper MB, Menachemi N. Factors associated with meaningful use incentives in children's hospitals. Pediatrics 2015;135(06):e1409-e1416

38 Veinot TC, Mitchell H, Ancker JS. Good intentions are not enough: how informatics interventions can worsen inequality. JAm Med Inform Assoc 2018;25(08):1080-1088

39 Sands DZ, Wald JS. Transforming health care delivery through consumer engagement, health data transparency, and patientgenerated health information. Yearb Med Inform 2014;9:170-176 